\title{
Cochlear Dead Regions in Adults and Children: Diagnosis and Clinical Implications
}

\author{
Brian C. J. Moore, Ph.D., ${ }^{1}$ and Alicja N. Malicka, Ph.D. ${ }^{2}$
}

\section{ABSTRACT}

The inner hair cells (IHCs) are the transducers of the cochlea; they convert mechanical vibrations to neural activity. When the IHCs and/or neurons are nonfunctioning over a certain region of the cochlea, this is referred to as a dead region. A dead region can be defined in terms of the characteristic frequencies of the IHCs and/or neurons immediately adjacent to the dead region. Dead regions can be detected, and their limits can be determined, using the threshold equalizing noise (TEN) test or by measurement of psychophysical tuning curves (PTCs). Both PTCs and the TEN test can be used to assess children as young as 7 years of age. The identification of dead regions can be helpful in determining the appropriate form of amplification. For both adults and children with restricted dead regions ("holes"), benefit is obtained from amplification of frequencies up to at least $4 \mathrm{kHz}$. For adults and children with extensive continuous dead regions starting at a relatively low frequency $(\leq 1.5 \mathrm{kHz}$ ) there may be little or no benefit from amplification of high frequencies.

KEYWORDS: dead region, TEN test, psychophysical tuning curve, amplification, hearing aid

Learning Outcomes: As a result of this activity, the participant will be able to (1) describe what a dead region is and describe two methods for diagnosing a dead region for adults and children, and (2) list the implications of dead regions for speech perception and the fitting of hearing aids.

\footnotetext{
${ }^{1}$ Department of Experimental Psychology, University of Cambridge, United Kingdom; ${ }^{2}$ School of Health and Rehabilitation Sciences, The University of Queensland, Queensland, Australia.

Address for correspondence and reprint requests: Brian C. J. Moore, Ph.D., Department of Experimental Psychology, University of Cambridge, Downing Street, Cambridge CB2 3EB, United Kingdom (e-mail: bcjm@cam.ac.uk).
}

Proceedings of the Widex Audiology Congress; Guest Editor, Robert Sweetow, Ph.D.

Semin Hear 2013;34:37-50. Copyright (C) 2013 by Thieme Medical Publishers, Inc., 333 Seventh Avenue, New York, NY 10001, USA. Tel: +1(212) 584-4662. DOI: http://dx.doi.org/10.1055/s-0032-1333150. ISSN 0734-0451. 
A dead region is a region in the cochlea where the inner hair cells (IHCs) and/or the auditory neurons are functioning very poorly, if at all. ${ }^{1-4} \mathrm{~A}$ sine wave that produces peak basilar membrane vibration in a dead region may not evoke sufficient neural activity within the dead region for that signal to be detected. However, if the signal is sufficiently intense, it may be detected via IHCs and neurons adjacent to the dead region. In other words, the signal may be detected via a place in the cochlea that is different from the place where that frequency would normally be detected. This is sometimes called "off-frequency listening" or "off-place listening." Psychoacoustic tests for diagnosing a dead region are basically tests for detecting when off-place listening is occurring. Hence, a dead region can be defined operationally as a region in the cochlea where the IHCs and/or neurons are functioning so poorly that a tone producing peak vibration in that region is detected by off-place listening. ${ }^{4}$

Using a frequency-to-place map, ${ }^{5-7}$ the boundary of a dead region can be defined in terms of the characteristic frequency of the IHCs and/or neurons immediately adjacent to the dead region. This is referred to as the edge frequency $(f e) .{ }^{1,3} \mathrm{We}$ can distinguish several types of dead regions:

1. A high-frequency (basal) dead region extends upward from $f e e^{2,8,9}$ When there is a dead region for all frequencies above $f e$, the dead region is referred to as continuous.

2. A low-frequency (apical) dead region extends downward from $f e{ }^{10,11}$ When there is a dead region for all frequencies below $f e$, the dead region is referred to as continuous.

3. A dead "hole" is a restricted dead region with lower and upper edge frequencies. ${ }^{12,13}$

4. There may be a continuous dead region below a lower $f e$ and above an upper $f e$, with a surviving "island" in between. ${ }^{14}$

5. There may be "patchy" dead regions (i.e., one or more dead holes with surviving regions in between).

\section{DIAGNOSIS OF DEAD REGIONS}

\section{Psychophysical Tuning Curves}

The "gold standard" for detecting dead regions is the psychophysical tuning curve (PTC). To measure a PTC, the sinusoidal signal is fixed in level, usually at a low sensation level such as 10$\mathrm{dB}$ sensation level. The masker is either a sinusoid or a narrowband noise. The masker level required just to mask the signal is measured as a function of the masker center frequency. For normally hearing subjects, the tip of the PTC (the frequency at which the masker level is lowest) usually lies close to the signal frequency. ${ }^{15-19}$ Thus, the masker is most effective when its center frequency is equal to the signal frequency. However, when hearing-impaired listeners are tested, PTCs have sometimes been found whose tips are shifted well away from the signal frequency. ${ }^{2,12,20-27}$ This happens when the signal frequency falls in a dead region. The masker is most effective when its center frequency coincides with the characteristic frequency at which the signal is being detected (via off-place listening). Thus, it is assumed that the tip of the PTC falls at the boundary of the dead region (i.e., at $f e$ ).

There are several problems associated with the measurement of PTCs. One is that the results may be affected by the detection of combination tones produced by the interaction of the signal and masker. ${ }^{12,25}$ This can happen especially for a person whose hearing at low frequencies is much better than at high frequencies. If the masker and the signal both fall in a region of hearing loss, they may interact to produce a simple difference tone that falls in a region of better hearing. This difference tone may be heard even when the signal itself is masked. To avoid this problem, Kluk and Moore $^{25}$ recommended adding a fixed lowpass noise to the main narrowband masker. The noise level is chosen so that the low-pass noise does not mask the signal, but it does mask any combination tone produced by the interaction of the signal and the masker. A second problem with PTCs is that the results can be influenced by the detection of beats between the signal and the masker, even when the masker is a narrowband noise. ${ }^{12,25}$ To avoid this problem, Kluk and Moore ${ }^{25}$ recommended the use of a relatively wide noise bandwidth, such as 300 Hz. Such a noise has inherent rapid amplitude fluctuations that make it difficult to detect beats between the signal and masker. However, the use of such a wide bandwidth is problematic for 
low signal frequencies, where the normal auditory filters are relatively narrow. ${ }^{28}$ When the noise bandwidth is larger than the auditoryfilter bandwidth, this can result in a broadening of the tip of the PTC, making it more difficult to determine the exact frequency at the tip. For low frequencies, a bandwidth of $160 \mathrm{~Hz}$ may be a good compromise. If precautions are not taken to prevent beats and combination tones from being used as a cue, PTCs with two or even three tips may occur, which makes the interpretation of the results very difficult. ${ }^{19,24,25,29}$

A practical problem for clinical applications is that the measurement of PTCs can be time consuming. The traditional method of obtaining PTCs is to measure the masker level required for threshold for several masker frequencies. Depending on how many masker frequencies are used and on the method that is used to estimate the masked threshold, it can take between 30 minutes and several hours to measure a PTC for a single signal frequency. A faster method is to use a masker that slowly sweeps in frequency, using a method resembling Békésy audiometry, to "track" the masker level required for threshold. ${ }^{24,30-32}$ The fast PTC method described by Sek et $\mathrm{al}^{31,32}$ requires about 3 minutes to measure a PTC. However, subjects sometimes require a few practice runs before they give stable results. ${ }^{26}$ Software for measuring fast PTCs can be downloaded from: http://hearing.psychol. cam.ac.uk.htm. The software runs on a PC with a good quality sound card.

\section{The Threshold Equalizing Noise Test}

The threshold equalizing noise (TEN) test was developed as a simple and quick method for diagnosing dead regions in the clinic. ${ }^{2,33}$ The test involves measuring the threshold for detecting a pure tone presented in a background noise called threshold equalizing noise. The signal level is adjusted to determine the masked threshold, while the TEN level is fixed. The noise spectrum is shaped in such a way that the threshold for detecting a pure tone in the noise is approximately the same over a wide range of tone frequencies for people with normal hearing and for people with hearing loss but without any dead regions. The masked threshold is approx- imately equal to the nominal level of the noise, specified as the level in a $132-\mathrm{Hz}$-wide band centered at $1000 \mathrm{~Hz}$. The value of $132 \mathrm{~Hz}$ corresponds to the equivalent rectangular bandwidth of the auditory filter, as determined using young, normally hearing listeners, which is denoted $\mathrm{ERB}_{\mathrm{N}} \cdot{ }^{34}$ When the signal frequency falls in a dead region, the signal will only be detected when it produces sufficient basilar-membrane vibration at a remote region in the cochlea where there are surviving IHCs and neurons. Because of the tuning of the basilar membrane, the amount of vibration at this remote region will be less than in the dead region, and so the noise will be very effective in masking it. Thus, the signal threshold is expected to be markedly higher than normal. A masked threshold that is $10 \mathrm{~dB}$ higher than normal is usually taken as indicating a dead region. ${ }^{2,33}$ For a positive diagnosis, the masked threshold of the signal in the TEN is also required to be $10 \mathrm{~dB}$ or more above the absolute threshold (i.e., the TEN must produce at least $10 \mathrm{~dB}$ of masking). If the TEN level/ERB $\mathrm{ER}_{\mathrm{N}}$ is set equal to or $10 \mathrm{~dB}$ above the absolute threshold at the test frequency, the second criterion is automatically satisfied when the first criterion is satisfied.

In the first version of the TEN test, ${ }^{2}$ all levels were calibrated in decibels sound pressure level (SPL) and the spectrum of the TEN was broad, so that the test could be used for a wide range of signal frequencies $(0.125 \mathrm{kHz}$ to 10 $\mathrm{kHz}$ ); this version is referred to as the TEN (SPL) test. In a later version of the test, ${ }^{33}$ all levels were calibrated in $\mathrm{dB}$ hearing level (HL) and the spectrum was made narrower to reduce the loudness of the TEN; this version is referred to as the TEN(HL) test. The TEN(HL) test can be used for signal frequencies in the range 0.5 to $4 \mathrm{kHz}$, and has to be used with specific headphones, namely Telephonics TDH39, TDH49 and TDH50 headphones (Telephonics; Farmingdale, New York). Recently, a version of the TEN test suitable for use with Etymotic Research ER-3A (Etymotic Research; Elk Grove Village, Illinois) insert earphones has been developed. ${ }^{35}$ This is called the TEN(ER3) test.

One drawback of the TEN test is that it does not give a precise estimate of the value of $f e$. If the criteria for diagnosis of a dead region are not met at a given test frequency but are met 
at the next higher test frequency, this suggests that the value of $f e$ lies somewhere between the two test frequencies. Thus the precision in estimating the value of $f e$ depends on the spacing of the test frequencies that are used. Also, the criteria for positive diagnosis of a dead region may not be met when the test frequency falls only a little above $f e$. Thus, in general, it is desirable to confirm the results of the TEN test, and to estimate the value of $f e$ more precisely, using PTCs.

\section{The Audiogram}

To assess whether dead regions could be diagnosed from the audiogram, Aazh and Moore ${ }^{36}$ and Vinay and Moore ${ }^{37}$ attempted to predict the presence of dead regions based on the audiogram. They compared the diagnosis with that obtained using the TEN(HL) test. ${ }^{33}$ They showed that it was not possible to achieve both high sensitivity and high specificity when attempting to predict the presence/ absence of a dead region from the audiogram. However, Vinay and Moore ${ }^{37}$ showed that, for each test frequency from 0.5 to $4 \mathrm{kHz}, 59 \%$ or more of ears had a dead region when the absolute threshold was above $70 \mathrm{~dB}$ HL. Thus, a hearing loss of $70 \mathrm{~dB}$ or more is suggestive of a dead region. However, a dead region can be present at a given test frequency when the audiometric threshold at that frequency is $55 \mathrm{~dB}$ or less, ${ }^{2,37,38}$ whereas a dead region may be absent when the hearing loss is $85 \mathrm{~dB}$ or more. ${ }^{2,37}$ Thus, there is a large range of uncertainty. A very steep slope of the audiogram, with thresholds worsening rapidly with increasing frequency, is suggestive of a highfrequency dead region but does not provide a reliable diagnostic method. ${ }^{37,39}$

\section{IMPLICATIONS OF DEAD REGIONS FOR THE CHOICE OF AMPLIFICATION CHARACTERISTICS FOR ADULTS}

There have long been reports suggesting that people with moderate to severe hearing loss at high frequencies often do not benefit from amplification of high frequencies, or even perform more poorly when high frequencies are amplified. ${ }^{40-45}$ It has been assumed in many of these studies that the subjects who did not benefit from amplification of high frequencies had reduced function or complete loss of function of IHCs and/or neurons, but no direct test for the presence of dead regions was conducted.

In two studies, adult subjects were tested for the presence of dead regions using both PTCs and the TEN test. ${ }^{8,9}$ All subjects had high-frequency hearing loss, but some had high-frequency dead regions and some did not. The dead regions, when present, appeared to be continuous rather than patchy (i.e., they extended from $f e$ upward, without any surviving "islands" of function). Also, the values of $f e$ were rather low, so the dead regions were extensive. Generally, the subjects with dead regions had more severe highfrequency hearing losses than those without dead regions. The speech stimuli were vowelconsonant-vowel nonsense syllables, using one of three vowels $(/ i /, \mid a /$, and $/ u /)$ and 21 different consonants. The stimuli were subjected to the frequency-gain characteristic prescribed by the "Cambridge" formula, ${ }^{46}$ which is roughly equivalent to a half-gain rule for midrange frequencies. The level of the input speech, before any amplification or filtering was applied, was $65 \mathrm{~dB}$ SPL.

Subjects were tested using broadband stimuli (upper frequency limit $7.5 \mathrm{kHz}$ ) and stimuli that were low-pass filtered with various cutoff frequencies. Changes in performance with cutoff frequency can be used to infer the extent to which information was being extracted in different frequency regions. In one experiment, ${ }^{8}$ the stimuli were presented in quiet. In another experiment, ${ }^{9}$ the stimuli were presented in steady speech-shaped noise, using a speechto-noise ratio chosen to give a moderate reduction in performance relative to that measured in quiet. The general pattern of results was similar for the two experiments, and we describe here only the results of the second experiment.

For subjects without dead regions, performance improved progressively with increasing cutoff frequency. This indicates that these subjects were able to make use of high-frequency information. The mean score for the broadband stimuli was $79 \%$. For subjects with dead regions, scores tended to increase with 
increasing cutoff frequency up to $1.5 f e$ to $2 f e$. For higher cutoff frequencies, scores tended to remain nearly constant or to decrease slightly. These results indicate that the subjects with dead regions extracted little or no information from frequencies that were well inside the dead regions. Generally, the best scores for the subjects with dead regions were well below those obtained by the subjects without dead regions when listening to broadband stimuli.

A study of Vestergaard ${ }^{47}$ provided data broadly consistent with the findings previously summarized. He tested experienced users of hearing aids. He used the TEN(SPL) test to diagnose dead regions. He also measured speech intelligibility using low-pass filtered word lists, while subjects listened through their own hearing aids, all of which provided substantial amplification at frequencies where there was hearing loss. To analyze his results, the ears tested were divided into those showing highfrequency dead regions with $f e$ in the range 0.75 to $1.5 \mathrm{kHz}$, and ears showing either no dead regions or dead regions with $f e$ at $3 \mathrm{kHz}$ or above. Ears showing evidence of dead "holes" were excluded from the analysis.

For the subjects with no dead regions or dead regions starting at $3 \mathrm{kHz}$ or above, performance tended to improve progressively with increasing low-pass filter cutoff frequency; the highest mean percent correct $(86 \%)$ was achieved for the widest bandwidth. For the subjects with $f e$ in the range 0.75 to $1.5 \mathrm{kHz}$, the pattern was less consistent, but the mean scores showed no clear trend with increasing cutoff frequency above $1 \mathrm{kHz}$, and the mean score for the widest bandwidth was only $58 \%$.

Overall, these results support the idea that subjects with extensive continuous high-frequency dead regions do not make as effective use of audible speech information at high frequencies as subjects without such dead regions. The subjects tested by Vestergaard ${ }^{47}$ all had experience using hearing aids that provided substantial high-frequency amplification. This suggests that the failure of the subjects with extensive high-frequency dead regions to benefit from the audibility of high frequencies was not due to lack of experience in hearing those frequencies.
Preminger et $\mathrm{al}^{39}$ investigated speech intelligibility and subjective hearing aid benefit using 49 experienced hearing aid users, with audiometric thresholds poorer than $50 \mathrm{~dB} \mathrm{HL}$ for at least two frequencies and no audiometric thresholds poorer than $80 \mathrm{~dB}$ HL. They used the TEN(SPL) test to diagnose dead regions, but using a 15- $\mathrm{dB}$ masked-threshold criterion rather than the $10-\mathrm{dB}$ criterion recommended by Moore et al. ${ }^{2}$ They measured speech intelligibility using two versions of the Quick SIN (signal in noise) test (Etymotic Research, Elk Grove Village, Illinois), one of which had increased gain above $1 \mathrm{kHz}$, with about $20 \mathrm{~dB}$ and $30 \mathrm{~dB}$ of gain at $2 \mathrm{kHz}$ and $3 \mathrm{kHz}$, respectively. Subjects with dead regions had poorer understanding of speech in noise than those without dead regions. However, both groups showed a benefit from increased high-frequency gain. It should be noted that the same frequency-gain characteristic was used for all participants, unlike the studies of Vickers et $\mathrm{al}^{8}$ and Baer et $\mathrm{al},{ }^{9}$ where the frequency-gain characteristic was tailored for each participant, based on their audiogram. The data of Preminger et al are not inconsistent with the idea that, for participants with extensive continuous dead regions, there is benefit from providing gain for frequencies up to about $1.7 \mathrm{fe}$, but no benefit from providing gain at higher frequencies.

In some studies conducted more recently, dead regions were diagnosed using the TEN (SPL) test ${ }^{48}$ or a modified version of the TEN (HL) test. ${ }^{49,50}$ The subjects tested in those studies mostly had less severe hearing loss than the subjects studied by Vickers et al, ${ }^{8}$ Baer et al, ${ }^{9}$ and Vestergaard. ${ }^{47}$ Also, the subjects had restricted dead regions (holes) rather than extensive continuous dead regions. The results generally showed that subjects both with and without dead regions benefited from the application of gain at high frequencies.

Overall, the results suggest that, for adult subjects with continuous high-frequency dead regions with $\mathrm{fe}$ at $1.5 \mathrm{kHz}$ or below, there is benefit from amplification of frequencies up to about $1.7 f e$, but no benefit of amplifying higher frequencies. However, for subjects with dead holes, patchy high-frequency dead regions, or continuous dead regions with $f e$ at $2 \mathrm{kHz}$ or above, amplification over the widest bandwidth possible may be most beneficial. 


\section{DIAGNOSIS OF DEAD REGIONS IN} CHILDREN

Malicka et $\mathrm{al}^{51}$ assessed whether it is possible to measure PTCs reliably for school-age children. They used a fast method, similar but not identical to the method of Sek and coworkers, as described earlier. ${ }^{31,32}$ Twelve normal-hearing children ( 7 to 10 years old) and five adults were tested. Fast PTCs were measured for 1and $4-\mathrm{kHz}$ signals. The center frequency of the narrowband noise masker either started below the signal frequency and was swept upward or started above the signal frequency and was swept downward. Both upward and downward sweeps were used for each subject and each signal frequency. The estimated tip frequency of the PTC tends to be above the signal frequency when the masker frequency sweeps upward and below the signal frequency when it sweeps downward. ${ }^{31,51}$ Measurements were repeated on a separate day to assess test-retest variability.

All children were able to perform the task. Examples of some of the PTCs are shown in Fig. 1. Results are shown for upward frequency sweeps for signal frequencies of $1 \mathrm{kHz}$ (top row) and $4 \mathrm{kHz}$ (bottom row). The fast PTCs were smoothed by taking a two-point moving average of the masker levels at the turn points, and the smoothed PTCs were used to estimate the frequency at the tip. The frequency at the tip was well defined for only $87 \%$ of the fast PTCs; for the other cases the PTCs had a flat region around the tip or had two or more tips. The variability in the estimated tip frequency was higher for the children than for the adults, but the difference was not statistically significant. The absolute value of the difference
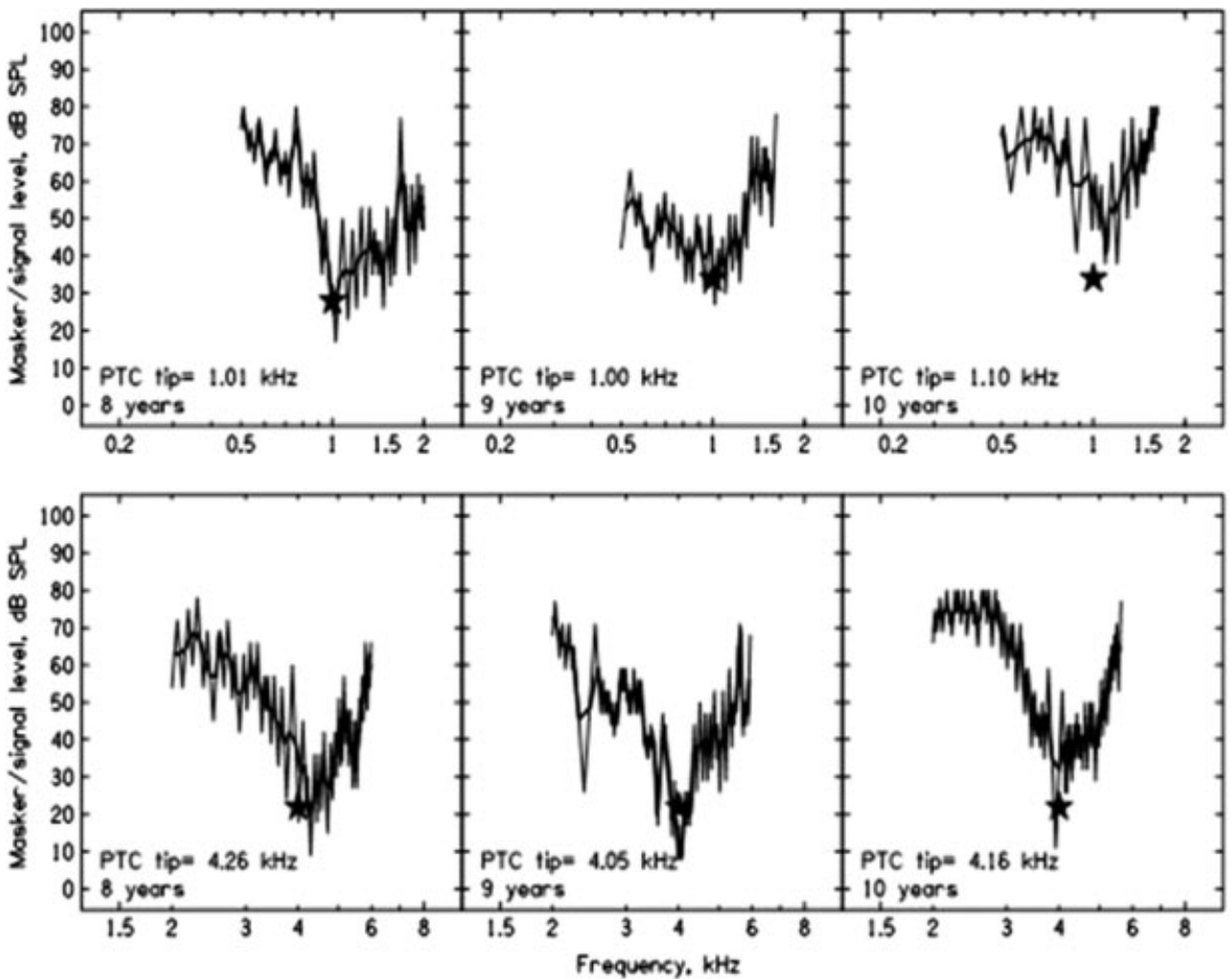

Figure 1 Examples of fast PTCs obtained from children with normal hearing. The age of the child is indicated in each panel. The top and bottom rows show results for signal frequencies of 1 and $4 \mathrm{kHz}$, respectively. The signal frequency and level for each PTC are indicated by the position of the filled star. The thin lines are the raw fast PTCs, and the thicker lines are two-point moving averages. The tip frequencies estimated from the moving averages are shown in each panel. The data are from Malicka et al. ${ }^{51}$ Abbreviations: PTC, psychophysical tuning curve; SPL, sound pressure level. 
between the estimated tip frequencies for test and retest was about $14 \%$ at $1 \mathrm{kHz}$ and $10 \%$ at $4 \mathrm{kHz}$. When the masker frequency was swept upward, the estimated tip frequency had a mean value that was shifted upward from the signal frequency by about $11 \%$ at $1 \mathrm{kHz}$ and $8 \%$ at $4 \mathrm{kHz}$. When the masker frequency was swept downward, the estimated tip frequency had a mean value that was shifted downward from the signal frequency by about $8 \%$ at $1 \mathrm{kHz}$ and $7 \%$ at $4 \mathrm{kHz}$.

There were only a few cases for children where the tip frequency was shifted down by more than $20 \%$ or up by more than $25 \%$, relative to the signal frequency. These values can therefore be used as limits that can be expected for most children without dead regions. Shifts in the tip frequency outside this range can be taken as indicating the presence of a dead region at the signal frequency.

Malicka et $\mathrm{al}^{27}$ assessed the extent of correspondence between results from the TEN(HL) test and fast PTCs. They also assessed whether the TEN(HL)-test criteria for diagnosing a dead region should differ for children and adults. They tested eight normalhearing children (16 ears) and 12 hearingimpaired children (21 ears), aged 7 to 13 years. For the normal-hearing children, masked thresholds in the TEN(HL) were measured for five TEN(HL) levels (30, 40, 50, 60, and $\left.70 \mathrm{~dB} / \mathrm{ERB}_{\mathrm{N}}\right)$. For the hearing-impaired children, the level of the TEN was selected sepa-

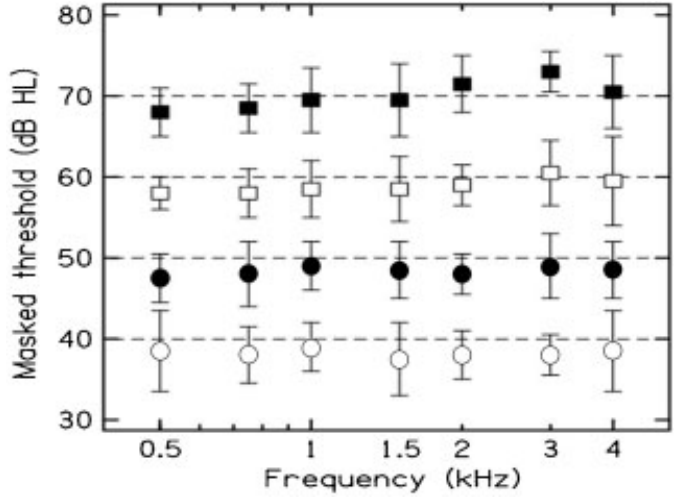

Figure 2 Mean TEN(HL) test results for normalhearing children for TEN(HL) levels of $40,50,60$, and $70 \mathrm{~dB} / \mathrm{ERB}_{\mathrm{N}}$. The data are from Malicka et al. ${ }^{27}$ Abbreviations: $d B$ hearing level $(H L) ; E R B_{N}$, equivalent rectangular bandwidth of the auditory filter, as determined using young, normally hearing listeners; TEN(HL), threshold equalizing noise with a spectrum chosen to produce equal masked thresholds in $\mathrm{dB}$ $\mathrm{HL}$.

rately for each ear based on the highest acceptable level minus $5 \mathrm{~dB}$.

The mean TEN(HL)-test results for the normal-hearing children for TEN(HL) levels of $40,50,60$ and $70 \mathrm{~dB} / \mathrm{ERBN}$ are shown in Fig. 2. The mean masked thresholds were usually slightly below the TEN level/ERB $\mathrm{E}_{\mathrm{N}}$ and were never more than $5 \mathrm{~dB}$ above it. The results resemble those found for adults. ${ }^{2,33,35}$

All hearing-impaired children were able to perform the TEN test and fast PTCs. Examples of PTCs for two hearing-impaired children are

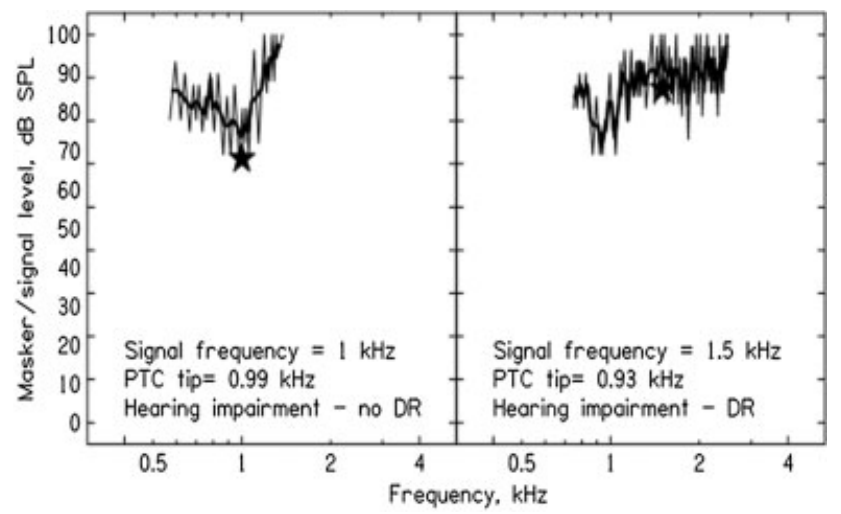

Figure 3 Examples of fast PTCs for two hearing-impaired children, one without a dead region at the signal frequency (left) and one with a dead region at the signal frequency (right). The thin lines are the raw fast PTCs, and the thicker lines are two-point moving averages. Abbreviations: DR, dead region; PTC, psychophysical tuning curve; SPL, sound pressure level. 
shown in Fig. 3. For the case shown in the left panel, the tip frequency of the PTC $(0.99 \mathrm{kHz})$ was close to the signal frequency $(1 \mathrm{kHz})$ indicating that there was no dead region at the signal frequency. For the case shown in the right panel, the tip frequency of the PTC (0.93 $\mathrm{kHz}$ ) was shifted well below the signal frequency $(1.5 \mathrm{kHz})$ indicating that there was a dead region at the signal frequency.

Examples of the correspondence between the PTCs and the TEN(HL)-test results are shown in Fig. 4. Eight ears showed no dead region on the TEN(HL) test and no dead region using PTCs. An example is shown in the leftmost column of Fig. 4. The masked threshold in the TEN (open squares) was always less than $10 \mathrm{~dB}$ above the TEN level of $60 \mathrm{~dB} / \mathrm{ERB}_{\mathrm{N}}$. The PTCs, determined using signal frequencies (filled symbols) of 1,2 , and $4 \mathrm{kHz}$, all had tips (open symbols) close to the signal frequency.

Nine ears showed a dead region on the TEN(HL) test that was confirmed using PTCs. An example is shown in the second column of Fig. 4. The TEN-test criteria for a dead region were met for signal frequencies of 0.5 , 0.75 , and $1 \mathrm{kHz}$, as indicated by the area with diagonal shading. For signal frequencies of $1.5 \mathrm{kHz}$ and above, the absolute threshold was too high for the TEN(HL) test to be performed (as indicated by the cross-hatched area), but it seems very likely that a dead region was present. The PTC determined using a signal frequency of $0.25 \mathrm{kHz}$ (middle panel)

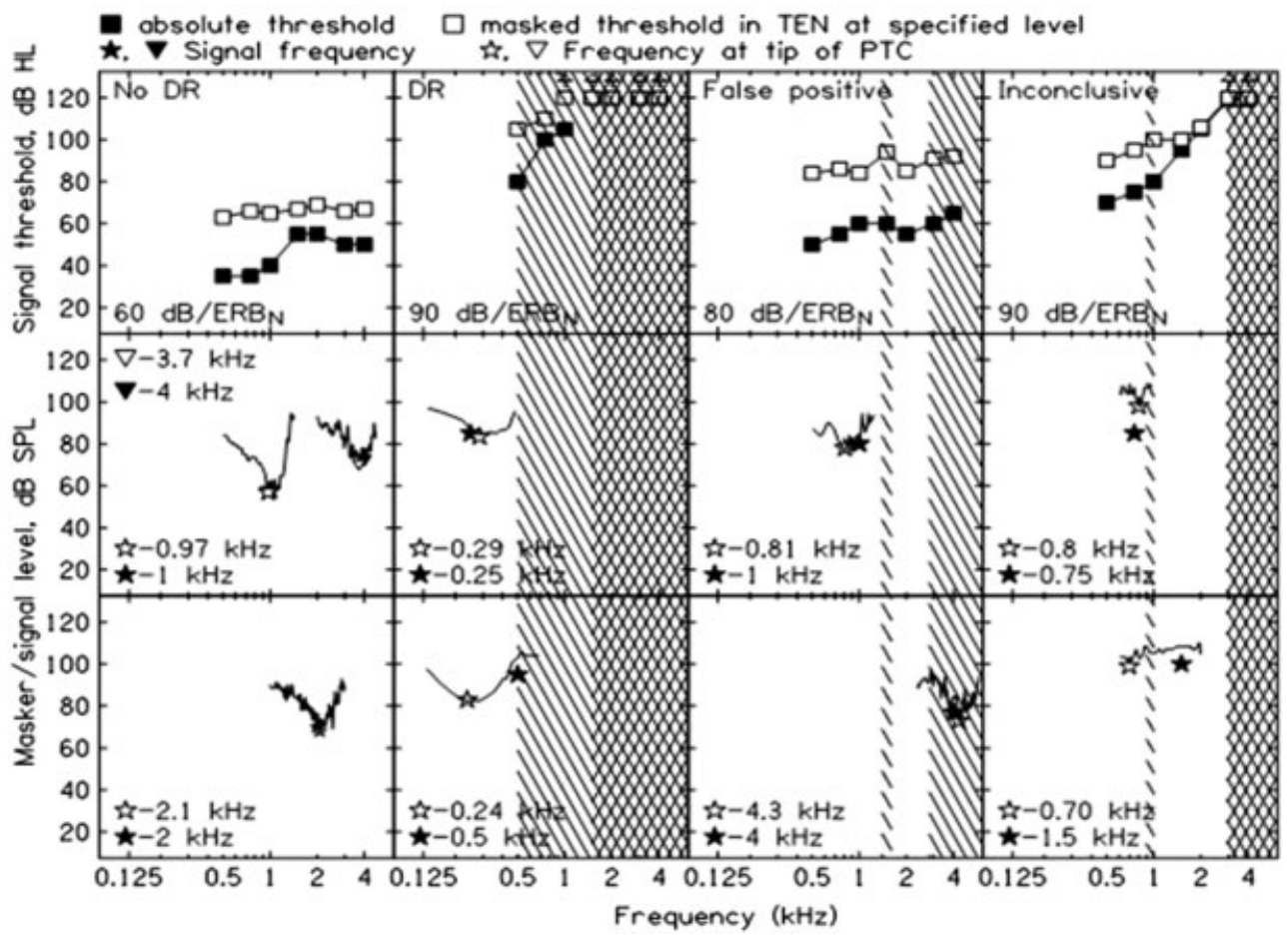

Figure 4 Examples of results from hearing-impaired children who were tested using the TEN(HL) test and fast PTCs. In each column, the top panel shows results for the TEN(HL) test. Filled squares show absolute thresholds and open squares show masked thresholds in the TEN(HL). The TEN(HL) level is indicated in each panel. Diagonally shaded areas indicate frequency regions where the TEN(HL) test results suggested the presence of a dead region. Cross-hatched areas indicate frequency regions where the absolute threshold was too high for the TEN(HL) test to be performed. The other panels show two-point moving averages of the fast PTCs. Solid symbols indicate the signal frequency, and open symbols indicate the estimated tip frequency of the PTC. The data are from Malicka et al. ${ }^{27}$ Abbreviations: ERB ${ }_{N}$, equivalent rectangular bandwidth of the auditory filter, as determined using young, normally hearing listeners; PTC, psychophysical tuning curves; SPL, sound pressure level; TEN(HL), threshold equalizing noise with a spectrum chosen to produce equal masked thresholds in $\mathrm{dB} \mathrm{HL}$. 
did not show a clearly shifted tip, whereas the PTC determined using a signal frequency of $0.5 \mathrm{kHz}$ (bottom panel) showed a downwardshifted tip.

Three ears showed a dead region on the TEN(HL) test that was not confirmed using PTCs; the PTCs did not show shifted tips when the signal frequency fell in a region identified as dead by the TEN(HL) test. An example is shown in the third column of Fig. 4. The findings for these three ears suggest that the TEN(HL) test can sometimes give falsepositive results with children. This also sometimes occurs with adults. ${ }^{2}$

For one ear, the TEN(HL)-test results suggested a restricted dead region (hole) around $1 \mathrm{kHz}$, and probably a dead region above $3 \mathrm{kHz}$. However, at $3 \mathrm{kHz}$ the absolute threshold was so high that the TEN(HL) did not raise the masked threshold above the absolute threshold, so the result must be regarded as inconclusive. These results are shown in the fourth column of Fig. 4. For a PTC measured with a signal frequency of $1.5 \mathrm{kHz}$ (bottom panel in the fourth column of Fig. 4), the PTC was very "flat," but the tip did appear to be shifted downward to about $0.7 \mathrm{kHz}$. This result might indicate that even the frequency region between 1 and $3 \mathrm{kHz}$ was dead. However, because the PTC is so flat, this result too must be regarded as inconclusive.

Overall, Malicka et $\mathrm{al}^{27}$ concluded that dead regions usually can be detected reliably in children using the fast-PTC technique and the TEN(HL) test. The most appropriate criteria for diagnosing a dead region using the TEN(HL) test are those recommended for adults. For cases where the masked threshold in the TEN(HL) is 10 to $15 \mathrm{~dB}$ above the TEN level/ERB $\mathrm{E}_{\mathrm{N}}$, the diagnosis of a dead region should be confirmed via measurement of fast PTCs, because false positives can occur when using the TEN(HL) test alone.

\section{BENEFIT OF AMPLIFICATION AT HIGH FREQUENCIES FOR CHILDREN WITH DEAD REGIONS}

Malicka et $\mathrm{a}^{52}$ investigated the benefit of highfrequency amplification for children with various degrees of high-frequency hearing loss, with and without dead regions. Dead regions were diagnosed using both the TEN(HL) test and fast PTCs, as described by Malicka et al. ${ }^{27}$ The children, aged 8 to 13 years, were divided into two groups according to the severity of their hearing loss. Group MS had moderate to severe loss (nine ears without dead regions and three ears with dead regions). For this group, the dead regions, when present, were restricted to the range 1.5 to $2 \mathrm{kHz}$. Group SP had severe to profound hearing loss (seven ears with highfrequency dead regions and one ear without a dead region). For this group, the dead regions, when present, were extensive and continuous, with estimated values of $f e$ between 0.75 and $1.5 \mathrm{kHz}$. The stimuli were vowel-consonantvowel nonsense syllables. They were subjected to the frequency-gain characteristic prescribed by the Desired Sensation Level fitting method $^{53}$ for $65 \mathrm{~dB}$ SPL speech, and presented via headphones. The gains provided were similar to those used in the children's own hearing aids. However, the children were fitted with multichannel compression hearing aids, whereas only linear amplification was used in the laboratory study.

The stimuli were either broadband or were low-pass filtered with various cutoff frequencies. For ears with a dead region, the cutoff frequencies were selected to cover the range from up to one octave below to well above the estimated value of $f e$. For ears without a dead region, the cutoff frequencies were chosen to be in the range between 1 and $4 \mathrm{kHz}$, usually 1,2 , and $4 \mathrm{kHz}$ and optionally 1.5 and $3 \mathrm{kHz}$. The number of conditions varied between four and six per ear.

The results for group MS are shown in Fig. 5. Consonant identification scores are plotted as a function of low-pass filter cutoff frequency. For all ears, the scores improved with increasing cutoff frequency up to $\sim 4 \mathrm{kHz}$ and tended to flatten above that frequency. There was a significant difference between scores for cutoff frequencies of 1 and $2 \mathrm{kHz}$, and 2 and $4 \mathrm{kHz}$, but there was no significant difference between scores for the cutoff frequency of $4 \mathrm{kHz}$ and the broadband condition. Thus, benefit was obtained for amplification of frequencies up to $4 \mathrm{kHz}$, but not for higher frequencies. 


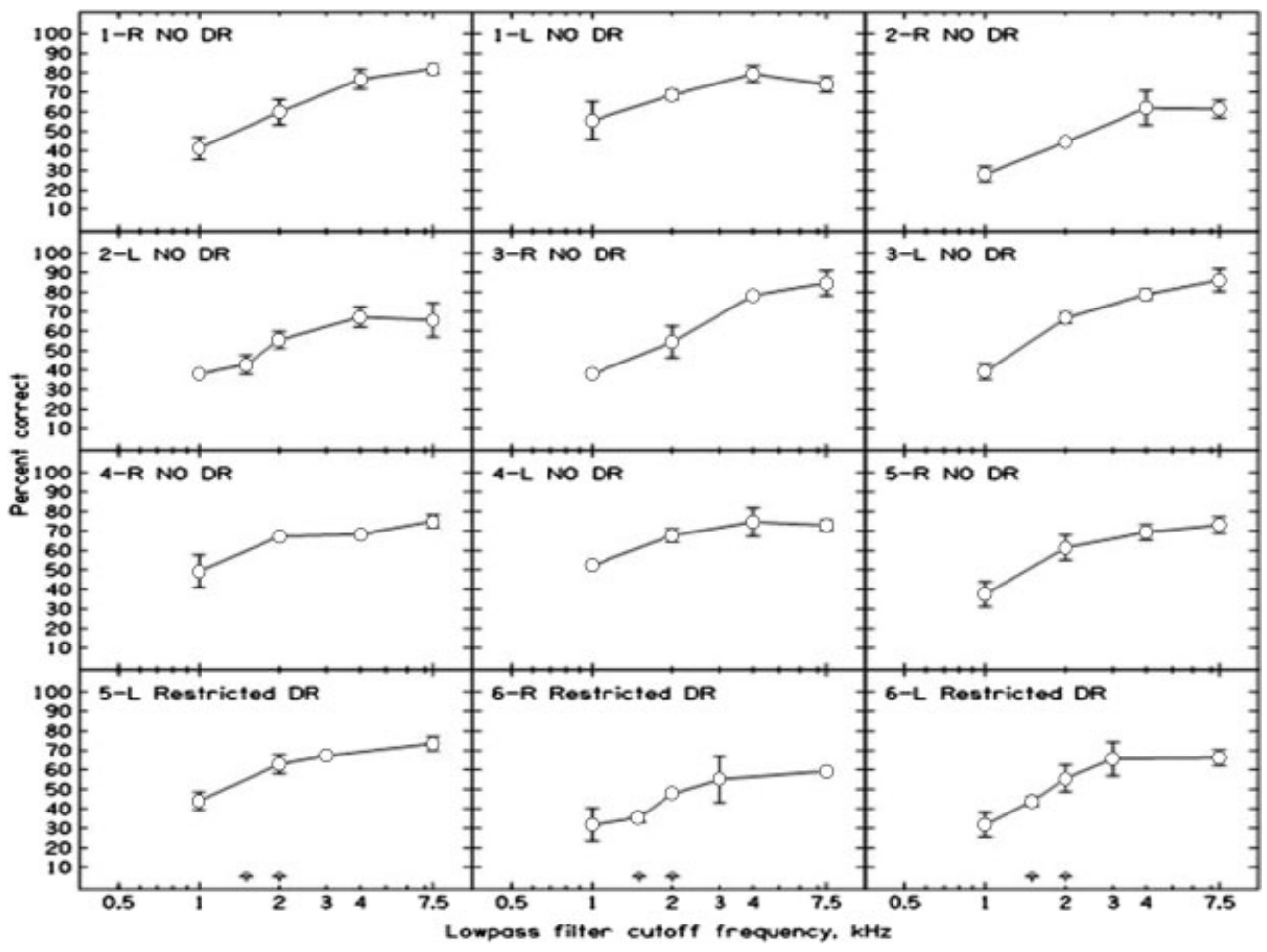

Figure 5 Consonant identification scores for individual subjects from group MS (group with moderate to severe loss), plotted as a function of low-pass filter cutoff frequency. Error bars represent \pm one standard deviation. The three upper rows show the results for the nine ears without dead regions, and the bottom row shows results for the three ears with restricted dead regions. The edges of the dead regions are indicated by the arrows close to the abscissa. The data are from Malicka et al. ${ }^{52}$ Abbreviation: DR, dead region.

The results for group SP are shown in Fig. 6. For the one ear without any dead region (7-L), performance was relatively poor, but it did improve with increasing cutoff frequency. Scores for the cutoff frequencies of 1,2 , and $4 \mathrm{kHz}$ were 33,39 , and $42 \%$, respectively. For the ears with dead regions, performance generally did not improve monotonically with increasing cutoff frequency. Mean scores for the ears with dead regions were 27,31 , and $30 \%$, for the cutoff frequencies of 1,2 , and $4 \mathrm{kHz}$, respectively. There was no significant effect of cutoff frequency across these three values. Thus the results provide no evidence for a benefit of presenting high-frequency speech components. For two ears (7-R and 11-L) performance worsened somewhat when the cutoff frequency was increased above $2 \mathrm{kHz}$.

To assess whether the changes in speech score with cutoff frequency were related to the values of $f e$, scores for speech low-pass filtered at $f e$ were compared with scores for speech that was low-pass filtered at one octave and two octaves above $f e$. The analysis was based on six ears (ear 8-L was excluded due to the large discrepancy between the frequency at the tip of the PTC, and the value of $f e$ estimated from the TEN(HL) test, leading to uncertainty as to the true $f e$ ). There was no significant effect of cutoff frequency. Thus, there was no benefit of amplified frequency components above $f e$.

Summers ${ }^{54}$ has argued that the most appropriate amplification for subjects with a severe sloping hearing loss can be selected based on the audiogram, and that a test for diagnosing dead regions is not required. He proposed, following common clinical practice, that amplification should be provided only for frequencies where hearing thresholds were better than $90 \mathrm{~dB}$ HL. However, this rule would not work 


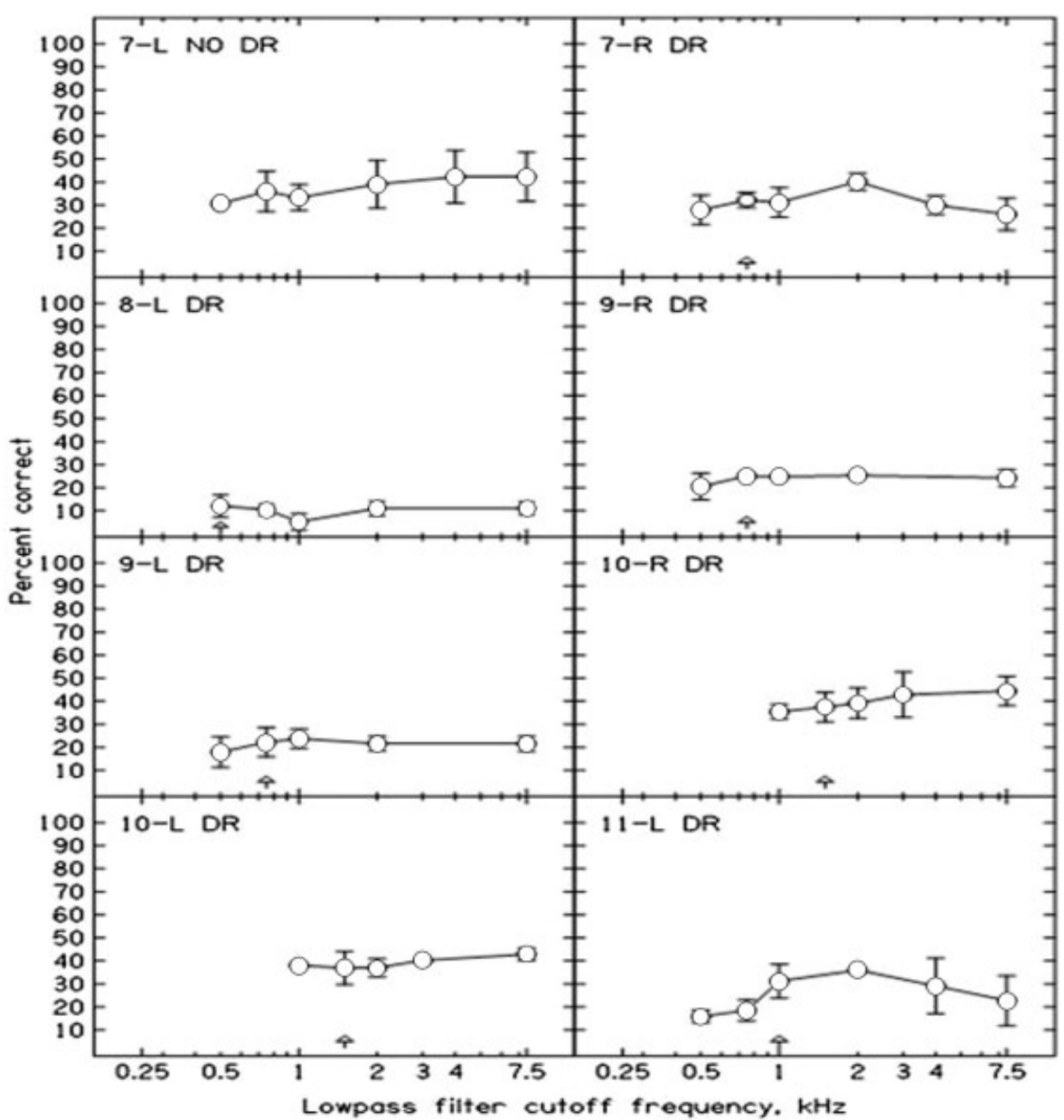

Figure 6 As Figure 4, but for group SP (with severe to profound loss). All ears except 7-L had extensive continuous dead regions with edge frequency at $1.5 \mathrm{kHz}$ or below. The data are from Malicka et al. ${ }^{52}$ Abbreviation: DR, dead region.

well for all of the children studied by Malicka et al. $^{52}$ For example, for ear 7-R (Fig. 6), Summer's rule would lead to no amplification for frequencies above $0.5 \mathrm{kHz}$, whereas 7 -R actually showed a benefit of amplifying frequencies up to $2 \mathrm{kHz}$.

The speech intelligibility index $(\mathrm{SII})^{55}$ was used to assess the effectiveness with which the children made use of audible speech components. The SII represents the proportion of the speech spectrum that is audible for a given speech material (with a weighting function representing the relative importance of different frequencies). The SII is calculated from the spectrum of the speech signal and the subject's hearing thresholds. The SII has a value between
0 (none of the speech signal is audible) and 1 (the speech signal is fully audible). The SII includes "corrections" to account for the selfmasking of speech and distortions that can occur at high listening levels. The SII values calculated by Malicka et $\mathrm{al}^{52}$ were based on the actual gains used for each ear.

To convert SII values to predicted speech scores, a transfer function relating scores to the SII needs to be estimated. The transfer function was derived by fitting a linear regression line to the results for children with hearing impairment but without dead regions. This line is shown in both panels of Fig. 7. The symbols in Fig. 7 show the percentage of correct responses for speech plotted against the 


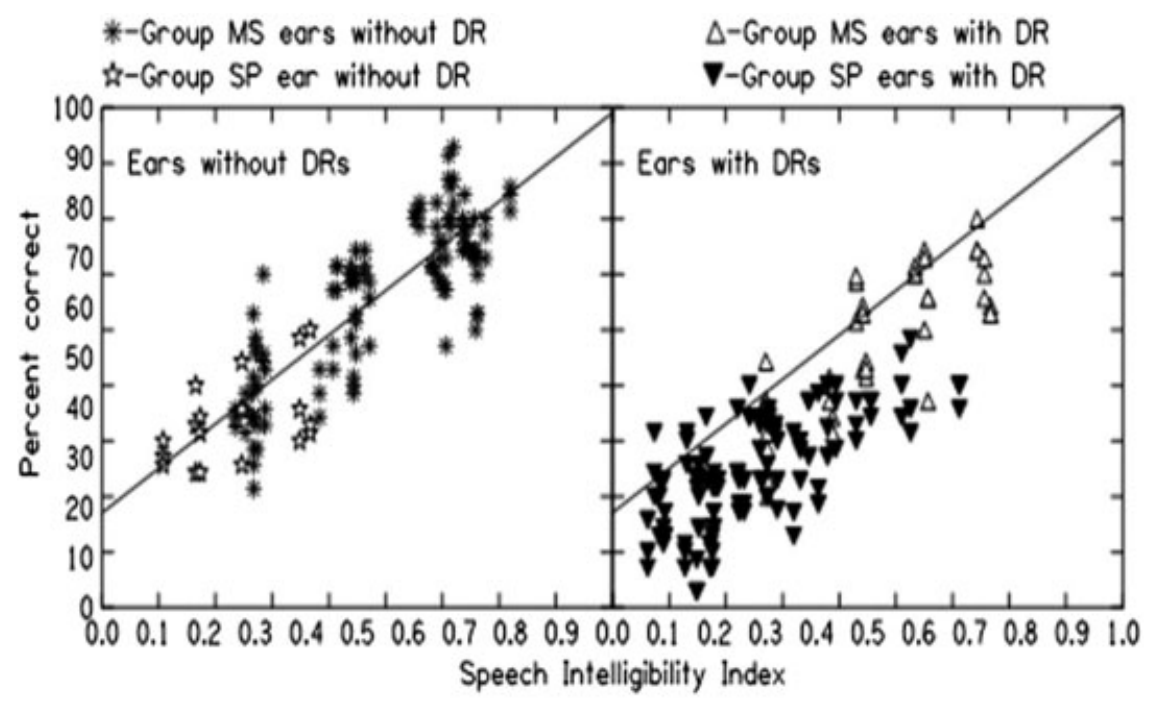

Figure 7 The symbols show percent correct scores for consonants plotted against the corresponding speech intelligibility index value. Each point represents one test condition and one ear. The left panel shows data for ears without dead regions (group MS, with moderate to profound loss asterisks; group SP, with severe to profound loss, open stars). The right panel shows data for ears with dead regions (group MS, open triangles; group SP, filled down-pointing triangles). The line in each panel is a linear regression line fitted to the data for all ears without dead regions. The data are from Malicka et al. ${ }^{52}$ Abbreviation: DR, dead region.

corresponding SII value. Each point represents one test condition and one ear. The left panel shows results for ears without dead regions (group MS—asterisks, group SP-open stars). The points are scattered roughly equally above and below the line, as expected. The scatter reflects individual differences in proficiency and random errors of measurement. It is noteworthy that the deviations of obtained scores from the line are similar for group MS and for the single participant in group SP who did not have a dead region.

The right panel of Fig. 7 shows the data for the ears with dead regions. Most of the points fall below the line, indicating that obtained speech scores were lower than predicted from the SII. This was especially the case for group $\mathrm{SP}$, who had continuous rather than restricted dead regions. The deviations from the line are greatest for higher values of the SII, which correspond to cases where the cutoff frequency was relatively high. The results indicate that the children with dead regions made less efficient use of audible speech components than the children without dead regions.

\section{CONCLUSIONS}

The results obtained with children are broadly consistent with those obtained using adults. Children with moderate to severe hearing loss, without dead regions, or with restricted dead regions, showed a benefit from the provision of amplification for frequencies up to $4 \mathrm{kHz}$. However, there was no significant difference in scores for the broadband condition and the condition with low-pass filtering at $4 \mathrm{kHz}$. For children with severe to profound hearing loss, those with extensive continuous dead regions showed reduced benefit from high-frequency amplification, and there was little benefit, or even a degradation, when amplification was provided for frequencies above $2 f e$. The single participant in group SP who did not have any dead region showed a benefit from providing amplification for frequencies up to 2 to $4 \mathrm{kHz}$.

The results may have important clinical implications for fitting hearing aids to children with severe to profound hearing impairment and extensive dead regions $(f e \leq 1.5 \mathrm{kHz}$ ). The results suggest that it may be appropriate to 
provide amplification only for frequencies up to about $2 f e$.

\section{ACKNOWLEDGMENTS}

The work of author B.M. was supported by the Medical Research Council (United Kingdom, grant G0701870).

\section{REFERENCES}

1. Moore BCJ, Glasberg BR. A model of loudness perception applied to cochlear hearing loss. Aud Neurosci 1997;3:289-311

2. Moore BCJ, Huss M, Vickers DA, Glasberg BR, Alcántara JI. A test for the diagnosis of dead regions in the cochlea. Br J Audiol 2000;34: 205-224

3. Moore BCJ. Dead regions in the cochlea: diagnosis, perceptual consequences, and implications for the fitting of hearing aids. Trends Amplif 2001;5:1-34

4. Moore BCJ. Dead regions in the cochlea: conceptual foundations, diagnosis, and clinical applications. Ear Hear 2004;25:98-116

5. Greenwood DD. Critical bandwidth and the frequency coordinates of the basilar membrane. J Acoust Soc Am 1961;33:1344-1356

6. Greenwood DD. A cochlear frequency-position function for several species-29 years later. J Acoust Soc Am 1990;87:2592-2605

7. Moore BCJ. Parallels between frequency selectivity measured psychophysically and in cochlear mechanics. Scand Audiol Suppl 1986;25(Suppl 25): 139-152

8. Vickers DA, Moore BCJ, Baer T. Effects of lowpass filtering on the intelligibility of speech in quiet for people with and without dead regions at high frequencies. J Acoust Soc Am 2001;110:1164-1175

9. Baer T, Moore BCJ, Kluk K. Effects of low pass filtering on the intelligibility of speech in noise for people with and without dead regions at high frequencies. J Acoust Soc Am 2002;112(3 Pt 1): 1133-1144

10. Vinay, Moore BCJ. Speech recognition as a function of highpass filter cutoff frequency for people with and without low-frequency cochlear dead regions. J Acoust Soc Am 2007;122:542-553

11. Vinay, Baer T, Moore BCJ. Speech recognition in noise as a function of highpass-filter cutoff frequency for people with and without low-frequency cochlear dead regions. J Acoust Soc Am 2008;123:606-609

12. Moore BCJ, Alcántara JI. The use of psychophysical tuning curves to explore dead regions in the cochlea. Ear Hear 2001;22:268-278
13. Shannon RV, Galvin JJ III, Baskent D. Holes in hearing. J Assoc Res Otolaryngol 2002;3:185-199

14. Vinay, Moore BCJ. Psychophysical tuning curves and recognition of highpass and lowpass filtered speech for a person with an inverted V-shaped audiogram (L). J Acoust Soc Am 2010;127:660-663

15. Chistovich LA. Frequency characteristics of masking effect. Biofizika 1957;2:743-755

16. Small AM. Pure-tone masking. J Acoust Soc Am 1959;31:1619-1625

17. Vogten LLM. Pure-tone masking: a new result from a new method. In: Zwicker E, Terhardt E, eds. Facts and Models in Hearing. Berlin, Germany: Springer-Verlag; 1974:142-155

18. Moore BCJ. Psychophysical tuning curves measured in simultaneous and forward masking. J Acoust Soc Am 1978;63:524-532

19. Kluk K, Moore BCJ. Factors affecting psychophysical tuning curves for normally hearing subjects. Hear Res 2004;194:118-134

20. Thornton AR, Abbas PJ. Low-frequency hearing loss: perception of filtered speech, psychophysical tuning curves, and masking. J Acoust Soc Am 1980;67:638-643

21. Florentine M, Houtsma AJM. Tuning curves and pitch matches in a listener with a unilateral, lowfrequency hearing loss. J Acoust Soc Am 1983; 73:961-965

22. Turner CW, Burns EM, Nelson DA. Pure tone pitch perception and low-frequency hearing loss. J Acoust Soc Am 1983;73:966-975

23. Moore BCJ, Vickers DA, Plack CJ, Oxenham AJ. Inter-relationship between different psychoacoustic measures assumed to be related to the cochlear active mechanism. J Acoust Soc Am 1999;106:2761-2778

24. Summers V, Molis MR, Müsch H, Walden BE, Surr RK, Cord MT. Identifying dead regions in the cochlea: psychophysical tuning curves and tone detection in threshold-equalizing noise. Ear Hear 2003;24:133-142

25. Kluk K, Moore BCJ. Factors affecting psychophysical tuning curves for hearing-impaired subjects with high-frequency dead regions. Hear Res 2005;200:115-131

26. Kluk K, Moore BCJ. Detecting dead regions using psychophysical tuning curves: a comparison of simultaneous and forward masking. Int J Audiol 2006;45:463-476

27. Malicka AN, Munro KJ, Baker RJ. Diagnosing cochlear dead regions in children. Ear Hear 2010;31:238-246

28. Glasberg BR, Moore BCJ. Derivation of auditory filter shapes from notched-noise data. Hear Res 1990;47:103-138

29. Hoekstra A, Ritsma RJ. Perceptive hearing loss and frequency selectivity. In: Evans EF, Wilson JP, eds. 
Psychophysics and Physiology of Hearing. London, England: Academic; 1977:263-271

30. Zwicker E. On the psychophysical equivalent of tuning curves. In: Zwicker E, Terhardt E, eds. Facts and Models in Hearing. Berlin, Germany: Springer-Verlag; 1974:132-140

31. Sek A, Alcántara JI, Moore BCJ, Kluk K, Wicher A. Development of a fast method for determining psychophysical tuning curves. Int $\mathrm{J}$ Audiol 2005;44:408-420

32. Sęk A, Moore BCJ. Implementation of a fast method for measuring psychophysical tuning curves. Int J Audiol 2011;50:237-242

33. Moore BCJ, Glasberg BR, Stone MA. New version of the TEN test with calibrations in dB HL. Ear Hear 2004;25:478-487

34. Moore BCJ. An Introduction to the Psychology of Hearing, 6th Ed. Bingley, UK: Emerald; 2012

35. Moore BCJ, Creeke S, Glasberg BR, Stone MA, Sek A. A version of the TEN Test for use with ER3A insert earphones. Ear Hear 2012;33:554-557

36. Aazh H, Moore BCJ. Dead regions in the cochlea at $4 \mathrm{kHz}$ in elderly adults: relation to absolute threshold, steepness of audiogram, and pure-tone average. J Am Acad Audiol 2007;18:97-106

37. Vinay, Moore BCJ. Prevalence of dead regions in subjects with sensorineural hearing loss. Ear Hear 2007;28:231-241

38. Huss M, Moore BCJ. Tone decay for hearingimpaired listeners with and without dead regions in the cochlea. J Acoust Soc Am 2003;114:3283-3294

39. Preminger JE, Carpenter R, Ziegler CH. A clinical perspective on cochlear dead regions: intelligibility of speech and subjective hearing aid benefit. J Am Acad Audiol 2005;16:600-613; quiz 631-632

40. Amos NE, Humes LE. The contribution of high frequencies to speech recognition in sensorineural hearing loss. In: Breebaart DJ, Houtsma AJM, Kohlrausch A, Prijs VF, Schoonhoven R, eds. Physiological and Psychophysical Bases of Auditory Function. Maastricht, The Netherlands: Shaker; 2001:437-444

41. Ching TY, Dillon H, Byrne D. Speech recognition of hearing-impaired listeners: predictions from audibility and the limited role of high-frequency amplification. J Acoust Soc Am 1998;103: 1128-1140

42. Hogan CA, Turner CW. High-frequency audibility: benefits for hearing-impaired listeners. J Acoust Soc Am 1998;104:432-441
43. Moore BCJ, Laurence RF, Wright D. Improvements in speech intelligibility in quiet and in noise produced by two-channel compression hearing aids. Br J Audiol 1985;19:175-187

44. Murray N, Byrne D. Performance of hearingimpaired and normal hearing listeners with various high-frequency cut-offs in hearing aids. Aust J Audiol 1986;8:21-28

45. Turner CW, Cummings KJ. Speech audibility for listeners with high-frequency hearing loss. Am J Audiol 1999;8:47-56

46. Moore BCJ, Glasberg BR. Use of a loudness model for hearing-aid fitting. I. Linear hearing aids. Br J Audiol 1998;32:317-335

47. Vestergaard MD. Dead regions in the cochlea: implications for speech recognition and applicability of articulation index theory. Int J Audiol 2003;42:249-261

48. Mackersie CL, Crocker TL, Davis RA. Limiting high-frequency hearing aid gain in listeners with and without suspected cochlear dead regions. J Am Acad Audiol 2004;15:498-507

49. Cox RM, Alexander GC, Johnson J, Rivera I. Cochlear dead regions in typical hearing aid candidates: prevalence and implications for use of high-frequency speech cues. Ear Hear 2011; 32:339-348

50. Cox RM, Johnson JA, Alexander GC. Implications of high-frequency cochlear dead regions for fitting hearing AIDS to adults with mild to moderately severe hearing loss. Ear Hear 2012;33:573-587

51. Malicka AN, Munro KJ, Baker RJ. Fast method for psychophysical tuning curve measurement in school-age children. Int J Audiol 2009;48:546-553

52. Malicka AN, Munro KJ, Baer T, Baker RJ, Moore BCJ. The effect of low-pass filtering on identification of nonsense syllables in quiet by school-age children with and without cochlear dead regions. Ear Hear 2013; In press

53. Cornelisse LE, Seewald RC, Jamieson DG. The input/output formula: a theoretical approach to the fitting of personal amplification devices. J Acoust Soc Am 1995;97:1854-1864

54. Summers V. Do tests for cochlear dead regions provide important information for fitting hearing aids? J Acoust Soc Am 2004;115:1420-1423

55. ANSI. ANSI S3.5-1997. Methods for the Calculation of the Speech Intelligibility Index. New York, NY: American National Standards Institute; 1997 